\title{
FACTORES RESILIENTES EN TRASTORNOS DE ALIMENTACIÓN EN GEMELOS
}

\section{RESILIENCE AND EATING DISORDERS IN TWINS}

\author{
ANALIA VERONICA LOSADA
}

MARTINA BISCUPOVICH ${ }^{\mathrm{i}}$

Recibido: 19 de julio de 2019

Aceptado: 1 de noviembre de 2019 



\title{
FACTORES RESILIENTES EN TRASTORNOS DE ALIMENTACIÓN EN GEMELOS
}

\section{RESILIENCE AND EATING DISORDERS IN TWINS}

Analia Veronica Losada

Martina Biscupovich

\begin{abstract}
Palabras clave: Gemelos, Trastornos Alimentarios, Resiliencia Keywords: Twins, Eating Disorders, Resilience
\end{abstract}

\section{RESUMEN}

El presente trabajo ha tenido por objeto estudiar cuáles son aquellos factores resilientes que podrían neutralizar el desarrollo de un trastorno de alimentación en gemelos. El tipo de estudio seleccionado ha sido de revisión bibliográfica. Los trastornos de la conducta alimentaria presentes en gemelos exhiben una tasa de concordancia que se sitúa en torno a más del cincuenta por ciento. Esto significa que en más de la mitad de los gemelos que comparten la misma dotación genética, cuando un gemelo desarrolla un trastorno alimentario el otro también lo padece. No es posible asegurar que los padres, a través de las vivencias que estos proporcionan, sean responsables de la individuación y desarrollo de sus hijos gemelos, pero sí pueden promover la constitución de una familia con características resilientes que cuente con la capacidad de generar 
determinados procesos, que le permitan enfrentar dificultades con mayor efectividad y a la vez salir fortalecidas de estas. La resiliencia individual y colectiva son complementarias, ya que la habilidad de enfrentar dificultades y salir airoso incluye respuestas que se puedan dar de manera individual y colectiva, frente al posible desarrollo de un trastorno de la conducta alimentaria.

\section{SUMMARY}

The present work has the intention of study object which are the resilient factors that could neutralize the development of a health problem in twins. The type of study selected was based on a literature review. Eating disorders present in twins exhibit an initial position-at-the-highest matching rate of fifty percent. This means that more than half of the twins who share the same genetic generation, when one twin develops an eating disorder the other also suffers the same. It is not possible to ensure that parents are responsible for the division and development of their children, but they can promote the constitution of a family with resilient characteristics that has the capacity to generate certain processes to cope. Individual and collective experience are complementary, because the ability to face difficulties and come out graceful includes responses that can be given individually and collectively, against the possible development of an eating disorder.

\section{INTRODUCCIÓN}

Esta investigación ha buscado ponderar los factores de resiliencia que podrían disminuir el desarrollo de un trastorno alimentario en gemelos, puesto que los miembros de embarazos múltiples presentan un riesgo mayor en el desarrollo de las patologías alimentarias.

Desde la perspectiva de Staudt, Rojo y Ojeda (2006), se señala que los trastornos de la conducta alimentaria son enfermedades de evolución crónica, que han tenido un gran aumento de su frecuencia en las últimas décadas y su etiología es multifactorial. Se caracterizan principalmente por el mantenimiento de un comportamiento distorsionado de la alimentación y una extrema preocupación por la imagen y el peso 
corporal. Dentro de los trastornos de la conducta alimentaria pueden encontrarse las anorexias, bulimias y los trastornos alimentarios especificados y no especificados. Estos trastornos exceden la afectación de la alimentación y la salud corporal, invadiendo el mundo mental de la paciente y conduciéndola al fracaso en sus responsabilidades personales, familiares, académicas, laborales. El malestar intenso y el sufrimiento psíquico, adopta de forma intensa la ansiedad y necesidad de mantener el control, sentimientos de culpa, baja autoestima, inestabilidad emocional y desesperanza; manifestándose a través del aislamiento, la desconfianza hacia los demás, el secreto y la ansiedad social. (Carol, 2010).

Existen estudios donde se analiza la relevancia que tienen los factores ambientales no compartidos en estos trastornos, con el fin de explicar por qué hermanos gemelos que han crecido en un ambiente familiar similar, se diferencian respecto a su conducta alimentaria, siendo patológica en unos casos mientras que en otros no. Entre los factores ambientales estudiados, Carol (2010), incluye el trato diferencial que pueda darse a los hermanos por parte de los padres, la personalidad y el temperamento de los sujetos, el estilo relacional de los sujetos, las situaciones estresantes vividas y por último las características diferenciales específicas. Losada (2011), expuso que la tasa de los trastornos de la conducta alimentaria entre gemelos pareció ser mayor que la observada en mellizos, $44 \%$ contra $12,5 \%$.

Coexiste entre los gemelos una notable semejanza en la estructura orgánica, en la predisposición a adquirir determinadas enfermedades, en el carácter, y en la inteligencia según Fernández García, Gálvez González y Villar Valdés (2010). La incidencia de los embarazos gemelares ha aumentado un $50 \%$ con respecto al siglo pasado debido al aumento de la edad materna y las técnicas de reproducción asistida (Durier, Vervaet y Gabriel, 2010). Cruz-Coke (1980), explica el fenómeno describiendo que los gemelos idénticos surgen a partir de la fecundación de un solo óvulo. Es por esta razón que antes de la implantación, sus individualidades ya están definidas por compartir el mismo componente genético. Shaffer (1999), amplía el concepto describiendo que un cigoto después de duplicarse se divide en células separadas pero idénticas, volviéndose luego en dos individuos. Se diferencian de los mellizos porque estos últimos son el resultado de dos óvulos genéticamente distintos, que han sido fecundados por dos espermatozoides distintos. (Ballard, Bricker, Reed, Wood, Neilson, Ballard, y Neilson, 2011).

Minuchin (1997), describe al subsistema fraterno como el primer laboratorio social en que los niños pueden 
experimentar relaciones con sus iguales. Para Trias, Ebeling y Moilanen (2012), el vínculo entre gemelos engendra un tipo de relación muy especial. Pueden ofrecerse de manera mutua apoyo, compañerismo y cariño, y se sugiere que es la relación más estrecha posible que se pueda dar entre dos personas. Por otra parte se enfrentan a sentimientos difíciles y en ocasiones, la identificación y la dependencia entre ellos puede ser muy marcada y fuerte. El desarrollo del sentido de sí, entre niños — descrito por Klein (2003) —, tiene particularidades, especialmente porque es necesario comprender cómo los dos niños hacen un manejo de su yo privado y su yo público, es decir el par. Todo dependerá de cómo la cultura colectiva y la cultura personal continuamente actúan, y en qué dirección se da esta actuación.

A raíz del carácter sociocultural de la gemelaridad y de los vínculos establecidos entre gemelo, co-gemelo y padres, Klein (2003) definió cuatro patrones de gemelaridad: la identidad única, la identidad interdependiente, la identidad dividida y la identidad individual; basándose en las prácticas educativas mediadas por los padres, así como posibles características de los niños y las niñas, consecuentes a estas prácticas de socialización.

El estudio Virginia Twin Study realizado en 1995 mencionado por Ro- dríguez Remón (2013), concluyó que la tasa de concordancia que se da entre gemelos y los trastornos de alimentación es de un 50-55\%. Por lo tanto, más de la mitad de los gemelos que comparten la misma dotación genética desarrollará un trastorno alimentario si el otro también lo padece.

Por tanto y dada la envergadura de la asociación entre trastornos de la conducta alimentaria y gemelazgos deben conocerse los factores que puedan neutralizar el desarrollo de esta patología. Los factores protectores se postulan como influencias que transforman, mejoran o alteran la respuesta de una persona a algún peligro que predispone a un resultado no adaptativo. Puede ser una cualidad o característica de una persona y no solo constituirse como una experiencia. Estos factores pueden agruparse en parentales, familiares, ambientales y en hereditarios o genéticos. (Losada, 2011; Losada, 2015).

La resiliencia se constituye como un factor protector a largo plazo. Losada (2011), define la resiliencia como aquella capacidad de los seres humanos que les permite sobreponerse a la adversidad y construir sobre ella. Tanto Quesada (2003) como Rutter (1999), postulan que la resiliencia abarca la diversidad individual de cómo las personas a lo largo del tiempo responden a los riesgos. Para 
Fraser (1997), el riesgo y la resiliencia forman un continuo, en donde cada constructo encarna el contrario del otro.

Dyer y McGuinnes (1996), generalizan la resiliencia como un concepto que está relacionado a varias características individuales y sistémicas. Grotberg (1995), entiende el concepto como la aptitud universal que le permite a una persona, una familia o un grupo minimizar o sobreponerse de las dificultades o anticiparse a adversidades inexcusables. Las conductas resilientes pueden ser: sostener la calma, mantener el desarrollo normal, o impulsar el crecimiento individual a pesar del presente que se esté atravesando.
En un primer momento, en el intento de encontrar las fuerzas protectoras, a raíz de lo escrito por Quesada (2003), la investigación se centró en las cualidades personales de los niños resilientes. Dichas cualidades son la autonomía y autoestima elevada. Más adelante, se arribó a la conclusión de que esa resiliencia derivaba también, en muchas ocasiones, de factores externos a los niños. Por ende, se identificaron los tres factores principales que son: los atributos del propio niño, las características de sus familias y las características del contexto social más amplio que rodea a niños y familias.

\section{OBJETIVO}

Estudiar la resiliencia como factor protector de los trastornos de la conduc- ta alimentaria bulimia y anorexia en gemelos adolescentes.

\section{METODOLOGÍA}

Se realizó un trabajo de revisión bibliográfica, en donde se analizó la bibliografía, tanto de origen nacional como extranjero considerando principalmente el continente americano, a fin de realizar un análisis crítico sobre la temática.

La metodología empleada es de estudio de revisión narrativa. Guirao
Goris, Olmedo Salas y Ferrer Ferrandis (2008), destacan que una investigación de revisión del tipo narrativa extrae información sobre una temática especificada, identificando los semblantes relevantes conocidos, los desconocidos y los controvertidos, explicitando lo investigado y presentando asociaciones. 
Se procedió en primera instancia a realizar la búsqueda del material a través de la Biblioteca de la Universidad Católica Argentina. Se analizó la bibliografía revisada y se presentó una perspectiva crítica sobre la misma. Se consultaron fuentes primarias como revistas científicas y de divulgación, libros, tesis de Licenciatura y Doctorado. Como fuentes secundarias se consultaron las bases de datos: Ebsco, Redalyc, Scielo y Psicothema. Se utilizaron palabras clave tales como: trastorno de alimentación, gemelos, factores de protección, resiliencia y en inglés: eatingdisordes, twins, resilience.

\section{TRASTORNOS DE LA CONDUCTA ALIMENTARIA}

Los trastornos de la conducta alimentaria han sido definidos por Carol (2010), como un conjunto complejo de cogniciones, actitudes y hábitos hacia la comida donde se enlazan con el peso y la imagen corporal. Estas ideas pueden convertirse en distorsiones que influyen en el funcionamiento cognitivo y afectan todos los aspectos del comportamiento individual, poniendo de manifiesto ciertas prácticas como la actividad física excesiva, conductas adictivas o conductas sociales particulares. Este trastorno genera elevadas consecuencias en diversas áreas del funcionamiento individual, biológico, psicológico y social.

La herencia tiene un rol esencial en la vulnerabilidad para desarrollar este tipo de trastorno. Se detectaron, en el plano endocrinológico, alteraciones hipotalámicas donde se encuentran aumentados los niveles de serotonina, alteraciones en el eje hipotálamo-hipófi- sis-tiroides y alteraciones del metabolismo de los hidratos de carbono y de la prolactina. Además, se han encontrado anomalías en la neurotransmisión cerebral. Es relevante estudiar si estas alteraciones son primarias o secundarias al desarrollo del trastorno, (Carol, 2010). Uno de los factores que está teniendo mayor importancia en la aparición y desarrollo de los trastornos de la conducta alimentaria, según Castro y Toro (2004), son los factores genéticos. Así pues, algunos estudios demuestran que las mujeres de familias en las que alguno de sus miembros tiene un trastorno de la conducta alimentaria tiene de siete a doce veces más probabilidades de padecerlo también. Del mismo modo, es relevante destacar que si bien la genética puede hacer que una persona sea más vulnerable a padecer estos trastornos, no por ello tiene por qué producirlo ni provocarlo obligatoriamente. Una per- 
sona genéticamente puede tener más predisposición que otra a desarrollar el trastorno y aun así no desarrollarlo, (Rodríguez Remón, 2013).

Los factores socioculturales se consideran un pilar relevante que influye en los trastornos de alimentación. El macro-contexto social está determinado por elementos como la moda, la publicidad, la información, las exigencias sociales, las relaciones de pareja y de amistad. A partir de estos elementos las personas desarrollan patrones de conducta para adaptarse a la sociedad, este tipo de adaptación puede ser susceptible a generar determinados comportamientos y actitudes no saludables. Carol (2010), propone que las presiones socioculturales de nuestra sociedad, el sobrepeso y la obesidad generan una alta insatisfacción corporal que está estrechamente relacionada al hincapié que hace la sociedad en poner la imagen como valor social. En consecuencia, se desencadenan conductas alimentarias negativas para contrarrestar la insatisfacción. Los mensajes difundidos por todo tipo de medios promueven la sobrevaloración del cuerpo delgado en el caso de las mujeres, relacionándolo directamente con el concepto de belleza y del cuerpo musculoso en caso de los hombres. Zamora, Martínez, Gazzo y Cordero (2002), agregan otros factores socioculturales que se potencian entre sí como las modas de vestir, las empresas de elaboración de productos y servicios para adelgazar, la progresiva exhibición del cuerpo, la publicidad, la influencia de los medios de comunicación, los cambios en el rol e imagen de la mujer en la sociedad y el rechazo psicosocial al sobrepeso. Coccaro (2012), constata que en los negocios de ropa, principalmente juveniles, los talles medianos son en realidad pequeños para el común de los compradores, imponiendo de esta forma un patrón de medidas que, difícilmente pueda ser alcanzado en la realidad, afectando la salud psicofísica principalmente de los adolescentes y jóvenes.

Coccaro (2012), sostiene que no es casual que estas patologías casi no se desarrollen en el mundo oriental en prevalencias similares, lo que da cuenta de que el factor cultural es un pilar esencial en estas afecciones.

En cuanto a los factores familiares Calvo (2002), señala que uno de los factores que puede influenciar a que un hijo desarrolle un trastorno de la conducta alimentaria es la trasmisión cultural que se da dentro de una familia sobre la importancia de la apariencia física. Si los padres dan excesivo valor al aspecto físico, es posible que les resulte difícil aceptar su cuerpo y por ende el de sus hijos e hijas y esto transmita el miedo de los hijos a engordar. González, Hidalgo, Hurtado, Nova, y Venegas (2011), señalan que en el seno 
familiar son riesgosos los comentarios reiterados sobre los cuerpos y siluetas de los adolescentes y determinadas patologías familiares de tipo interaccional.

Vázquez-Arévalo, Mancilla-Díaz, López-Aguilar, Álvarez-Rayón, y Tena-Suck (2010), señalan que los factores familiares son relevantes ya que la familia es el primer grupo de pertenencia donde se transmiten actitudes y creencias y se modelan conductas alimentarias que incorporará el sujeto a lo largo de su vida. La familia puede propiciar la aparición de tales trastornos, puede mantenerlos, en cuanto a que sus interrelaciones permitan que los síntomas persistan a través del tiempo o puede desarrollar un papel protector brindando a sus miembros las habilidades para superar o evitar los factores de riesgo. Las familias afectadas presentan menor flexibilidad, cohesión, comunicación intrafamiliar, expresión emocional, mayor rechazo y sobreprotección paterna. Los pacientes perciben que en su familia se les da escasa importancia a las dimensiones del desarrollo como la autonomía y las actividades culturales y sociales. El ambiente lo describen deteriorado y existe la presencia de sentimientos negativos entre los familiares. El funcionamiento familiar se ha distinguido de acuerdo con el tipo de trastorno. Los pacientes con anorexia señalaron mayor insatisfacción con su vida familiar, altos niveles de crítica y hostilidad, dificultad en la solución de problemas y rigidez. Los pacientes con bulimia perciben menos apoyo e independencia y mayor desintegración al presentarse en sus familias actitudes de negativismo y hostilidad frente a sus necesidades emocionales.

Vázquez-Arévalo et al. (2010), describieron los factores del funcionamiento familiar que participan como protectores y predictores de los trastornos de la conducta alimentaria. El factor más relevante que explicó el riesgo del desarrollo del trastorno es la expresión en los grupos. El dejar de compartir sentimientos y emociones dentro de las familias deja al descubierto necesidades básicas como la aceptación y el afecto. Otro factor de riesgo resultó ser el de organización, entendiéndose como la falta de planificación en las actividades cotidianas y la carencia de asignación de las responsabilidades familiares. Por otro lado, los factores protectores para todos los grupos resultaron ser el de la actuación y el factor intelectual-cultural. La actuación representa el grado en que las actividades, tales como el colegio o el trabajo se enmarcan en una estructura orientada a la acción-competencia. Y el factor intelectual-cultural, se refiere al interés en actividades sociales, políticas, culturales e intelectuales.

Carol (2010), afirma que uno de los principales factores psicológicos 
que está asociado a los trastornos de alimentación es la distorsión de la imagen corporal. Es un constructo multidimensional que representa cómo los individuos a través de las evaluaciones generales que hacen de su cuerpo piensan, sienten y se comportan. Otros factores psicológicos asociados son la insatisfacción, los índices de masa corporal extremos, la baja autoestima, el afecto negativo, la depresión, la ansiedad y el uso y abuso de dietas injustificadas que preparan el terreno para el desarrollo, arraigo y agravación de estas enfermedades.

Ciertos rasgos de personalidad también conforman un papel muy importante para el desarrollo y el mantenimiento de estos trastornos como el perfeccionismo, la baja autoestima, el retraimiento social y la excesiva rigidez. Zamora (et al., 2002), agregan que hay otras circunstancias además de los estados de ansiedad y depresión que producen insatisfacción corporal y adelgazamiento. Dentro de ellas tenemos la baja estatura, el embarazo, el vegetarianismo, dismorfofobias, accidentes con alguna consecuencia deformante y trastornos obsesivos. El factor de riesgo aumentará cuando existen perturbaciones de la personalidad no resueltas de la niñez y que se agravan en la adolescencia, reflejándose en estos trastornos que surgen como la continuación de dificultades que no fueron resueltas.
Son considerados también factores de riesgo el sexo femenino, la situación de pubertad, la personalidad previa, el sobrepeso u obesidad y la actividad física excesiva. (Zamora et al., 2002). Los factores pueden clasificarse en tres categorías: predisponentes, precipitantes y perpetuantes. Los factores predisponentes se constituyen como ser mujer en un país industrializado, herencia, historia familiar de trastornos alimentarios, no poder comunicar emociones negativas con facilidad, ser perfeccionista, ser complaciente con los demás, tener baja autoestima, tener padres sobreprotectores, no tener habilidades para resolver conflictos, sentirse presionada por el medio, vivir experiencias adversas, alteraciones en neurotransmisores como la serotonina, hacer dieta asociada a presiones individuales, interpersonales y socioculturales. Los factores precipitantes son la dinámica familiar y los comentarios negativos del círculo íntimo como la familia o amigos, los eventos negativos como un fracaso académico, conflictos familiares o pérdidas afectivas, hacer dieta como factor necesario, ser víctima de abuso sexual y tener dificultades en el desarrollo de la transición a la adultez. Por último, los factores perpetuantes pueden ser los procesos psicológicos o los procesos biológicos que estén relacionados con la desnutrición y la realimentación, como cambios en el sistema 
endocrino, en tasas metabólicas o en la función gastrointestinal. (Coccaro, 2012).

Los trastornos de la conducta alimentaria invaden el mundo mental de la paciente y la conduce al fracaso en las responsabilidades personales, familiares, académicas, laborales, ya que se va generando una situación de malestar intenso y sufrimiento psíquico el cual adopta de forma intensa la ansiedad y necesidad de mantener el control, sentimientos de culpa, baja autoestima, inestabilidad emocional, desesperanza, aislamiento y desconfianza hacia los demás. (Carol 2010). Es por esta razón que Herscovici y Bay (1990), sostienen que los trastornos de la conducta alimentaria requieren de intervenciones multidisciplinares, por la complejidad y las múltiples consecuencias que afectan a las personas que lo padecen.

\section{HERMANOS}

Minuchin (1997), describe al subsistema fraterno como el primer laboratorio social en que los niños pueden experimentar relaciones con sus iguales. En este, los niños se apoyan y aprenden a lograr amigos y aliados, a negociar, cooperar, compartir, y a lograr reconocimiento por sus habilidades.

Kancyper (2004), también se ocupó del estudio del complejo fraterno y lo definió como: "un conjunto organizado de deseos hostiles y amorosos que el niño experimenta respecto de sus hermanos" (p. 243). El complejo fraterno cumple con cuatro funciones estrechamente relacionadas entre sí. La primera función es la función sustitutiva, la función defensiva, la función elaborativa y la función estructurante.

Dentro del subsistema fraterno, Bank y Kahn (1988), reconocen ocho pro- cesos de identificación que se dan entre hermanos, estos pueden ser transitorios o pueden durar toda la vida. Se dividen en tres categorías: la identificación estrecha, que se da cuando ambos hermanos sienten que hay entre ellos una gran semejanza y muy pocas diferencias; la identificación parcial cuando ambos hermanos sienten que hay entre ellos algunas semejanzas y alguna diferencia. Y por último, la identificación distante en la que ambos hermanos sienten que hay entre ellos una gran diferencia y muy poca semejanza. En el caso de la identificación estrecha, existen tres modelos. El primer modelo es el de gemelazgo, en donde se genera una relación de unificación, cuando en la familia no se definen en forma nítida los roles e identificaciones de los niños, existe el riesgo de fusión de identidades, el niño se ve como 
el doble del otro. Si los padres no están atentos a las necesidades y atributos individuales de los niños, los hermanos pueden llegar a una forma extrema de indiferenciación. Funcionan como si el otro hermano fuera un apéndice, un alter ego cuya presencia constante resulta imprescindible, este caso es más común en mellizos. El segundo modelo es el de fusión, en donde se genera una relación de confusión, que puede ocurrir cuando los padres no definen de modo nítido la identidad del niño. Ninguno de los hermanos posee la confianza sufici- ente como para desprenderse del otro, ya que ambos dudan de sus identidades personales. Por último, el tercer modelo de idealización trata sobre el culto del héroe, es decir, un proceso unilateral que parte del hermano menor y se dirige al hermano mayor. Un niño puede idealizar al hermano quien posee características positivas o negativas, y desea imitar esos rasgos valiosos. Esto representa idealizar a otro e imitarlo nos proporciona la identidad que desearíamos que se desarrolle en nosotros.

\section{GEMELOS}

La gestación de dos o más fetos se denomina embarazo múltiple, con estados concomitantes con la condición materna, gestación y el parto, y es considerado de alto riesgo para la salud perinatal (Sánchez Ramírez, Carrillo Bermúdez, Sanabria Arias y Olivella Mejía, 2019).

El término gemelo proviene del latín gemellus y expresa "partido en dos". (Eddleman, Stone, Lynch y Berkowitz, 2002; Sánchez Ramírez, Carrillo Bermúdez, Sanabria Arias y Olivella Mejía, 2019).

La gestación múltiple es una de las temáticas de interés en la psicología perinatal y en la obstetricia, reflejando acciones multidisciplinarias en el marco de la promoción de la salud y la prevención. (Endres, Wilkins, 2005; Wimalasundera, 2010).

Los gemelos idénticos surgen por haber sido creados de un solo óvulo y un único espermatozoide. Es por esta razón que antes de la implantación sus individualidades ya están definidas por compartir el mismo componente genético. (Cruz-Coke, 1980). Shaffer (1999), amplía el concepto describiendo que un cigoto comienza a duplicarse y se divide en células separadas pero idénticas, las cuales luego se vuelven dos individuos. La circunstancia de compartir un genotipo es la razón por la cual existen gemelos monocigoóticos. A raíz de que com- 
parten la misma información genética, Gromada y Hurlburt (2006), afirman que por eso los gemelos idénticos son del mismo sexo, y tanto su temperamento como su apariencia física suelen ser muy parecidos. En algunos casos, los gemelos pueden compartir la placenta, o pueden tener cada uno su placenta. Se diferencian de los mellizos porque estos son el resultado de dos óvulos genéticamente distintos, que han sido fecundados por dos espermatozoides distintos, por eso pueden ser de diferente sexo. Comparten estadísticamente el $50 \%$ de sus genes por eso su grado de identidad genética es igual que dos hermanos nacidos de gestaciones separadas. (Ballard et al., 2011).

La similitud que existe entre los hermanos gemelos ha sido señalada en muchas de sus facetas como algo asombroso. Existe también entre ellos una notable semejanza en la estructura orgánica, en la predisposición a adquirir determinadas enfermedades, en el carácter, en la inteligencia, llegando a presentar incluso las mismas huellas dactilares. (Fernández García, Gálvez González y Villar Valdés, 2014).

La incidencia de los embarazos gemelares se encuentra en una escalada ascendente debido al aumento de la edad materna y las técnicas de reproducción asistida. Aproximadamente el $50 \%$ de los gemelos nace antes de las
37 semanas y el 6-10\% nace antes de las 32 semanas y su peso es menor a 1.500 gramos. (Durier, Vervaet y Gabriel, 2010). Mares y Casanueva (2001), coinciden en la incrementación de este tipo de embarazos en donde la gestación múltiple con mayor frecuencia es la gemelar doble y en menor grado los embarazos de trillizos o cuatrillizos. La frecuencia global de gemelaridad es de 0,93 a $1 \%$ nacimientos. (Laterra, Susacasa, Frailuna, Flores, y Secondi, 2006).

La condición de ser mellizo tiene un potencial traumático en la medida en que el niño y sus padres no lo puedan tramitar correctamente, manifestándose en conductas particulares entre los hermanos y en la dinámica con los padres. Trias, Ebeling y Moilanen (2012), explican que el vínculo entre gemelos engendra una relación estrecha, apoyo, compañerismo y cariño, y a la vez se enfrentan a sentimientos difíciles, bajo identificación y dependencia muy fuerte. Coexiste la fantasía de que existe un solo espacio, un solo tiempo y una sola posibilidad para dos. Según esta fantasía, por ejemplo, existiría una sola carrera profesional, una sola posición económica y una belleza excluyente. Se puede llegar a dar que en una relación un hermano sea dominado por el otro. El dominante tiene mayor necesidad de ese otro y es frecuente que eso le resulte inaguantable y en cambio el dominado puede desprenderse del 
dominante, acomodándose a tal situación.

Perlman (2004), destaca que los gemelos pueden expresar diversas ideas sobre la gemelaridad y la individualidad. Algunos revelan que sienten que si están separados son menos que un ser humano completo, y que si están juntos son un ser humano inusualmente poderoso. Suele ser frecuente el tema del gemelo bueno y el gemelo malo como si al independizarse uno se transformara en la materialización de lo bueno y el otro de lo malo. Algunos caracterizan a su gemelo como el otro lado de sí mismos. Otros expresan que cuando quieren estar con ellos mismos, quieren decir en realidad, que quieren estar con su gemelo. Un temor expresado por algunos gemelos es que sus padres confundan sus identidades como si fueran recién nacidos, y que no sepan diferenciar quién es quién. La separación y diferencia entre uno u otro es un proceso que evoluciona de manera muy lenta.

La manera en la que un gemelo se moviliza desde una posición de nosotros a una posición de yo es arduo trabajo de la niñez y continúa en la adultez. Si este proceso ya es dificultoso para cualquier ser humano, será aún más para los gemelos. Sobre esto Mahler, Pine y Bergman (1977), describen que alrededor del sexto mes de vida un bebé comienza con los intentos de separación e individuación, explorando a la madre e intentando pasar de la situación inactiva a una mayor independencia. Cuando el bebé llega a individualizarse lo suficiente como para registrar el rostro de su mamá, controla y compara los rasgos de los otros. A partir de repetidas interacciones de un bebé con el medio que lo rodea, va a ir descubriendo quién es a través de la separación de él mismo con el resto del entorno. La primera separación es con la madre, este proceso se denomina temor a lo desconocido. En donde los padres perciben que el bebé que antes se dejaba alzar por todo el mundo, ahora no lo hace más. En el caso de gemelos, el proceso se hace más difícil por tener un compañero constante que es idéntico, del cual deben aprender a distinguirse. Y en la medida que la apariencia sea exacta, el proceso es ampliamente más difícil. En el inicio de esta etapa de separación e individualización, la presencia de alguien tan similar a uno mismo se desarrollaría de cierta manera perturbadora. Descubrieron que es frecuente que los gemelos de entre seis y diez meses parecen percibirse el uno al otro, sin embargo, se pueden mostrar reacios a mirarse. (Perlman 2004). Al final del primer año de vida y primeros meses del segundo, se presenta de manera separada el proceso intrapsíquico de separación individuación. El camino de la separación continúa el recorrido de la 
diferenciación, la formación de límites, el distanciamiento y la desvinculación de la madre. Paralelamente, el camino de la individuación consta del desarrollo de la percepción, la cognición, la prueba de realidad, la memoria y la evolución de la autonomía intrapsíquica. Los logros que se alcanzan por estos procesos evolutivos son poder diferenciarse corporalmente con la madre, establecer un vínculo particular con ella y desarrollar los aparatos autónomos del yo en cercanía con la mamá. (Mahler et al., 1997).

El proceso de organización de sí mismos en los gemelos es más difícil por la particular relación que establecen entre ellos. Estos niños tienen que reconocerse y distinguirse entre sí. La construcción de la imagen de sí mismo está entrelazada por la separación y por la distinción del propio gemelo. La separación es la representación del propio espacio y ser consciente de tener un cuerpo proporcionado de grosor. La distinción se refiere a la conciencia de la propia individualidad que tiene singularidades concretas. (Mahler et al., 1997).

Para un progreso adecuado de la personalidad en cada gemelo, el sentimiento de su parecido con el co-gemelo no tendría que obstaculizar la construcción de una identidad personal integrada. Cerruti, Prino y Quaglia (2008), exponen que la similitud física interfiere aún más en la construcción de la imagen de los gemelos, haciéndolo al proceso de separación-individuación mucho más complejo, en especial por la proximidad advertida con el otro. Como siempre compartieron el ambiente desde el útero materno, se les hace difícil vivir como propio el espacio que su cuerpo habita. A raíz de esto los gemelos desarrollan una percepción distinta de las fronteras de sí mismo en comparación con los niños no gemelos porque cada movimiento es asociado y consecutivo al del co-gemelo.

A través de la imitación gráfica, se puede delimitar el nivel de homogeneidad o de heterogeneidad anhelado por el niño respecto al otro. La necesidad de diferenciarse del otro se ha analizado según las diferencias de los dibujos. Los datos reunidos por Cerruti et al. (2008), muestran comportamientos gráficos diferentes, los mellizos se dibujan mucho más parecidos entre ellos, muestran la tendencia a uniformar las figuras o incluso dibujarlas idénticas. Antagónicamente los gemelos cuando dibujan las dos figuras tienden a agregar un detalle que especifique la diversidad y la identificación de sí mismo respecto al otro. La necesidad del reconocimiento urgente por parte del dibujante como por parte de los demás se manifiesta como algo prioritario en relación con la identidad de pareja que se ve expresado en la tendencia habitual de los gemelos en 
hacer que cada figura se reconozca por tener elementos de distinción, pudiendo entenderse como una necesidad de individuación considerada esencial en el proceso evolutivo. Los datos del estudio plantean que además de los procesos imitativos e identificativos aparecen también procesos inclinados a la diferenciación, ambos vitales y cruciales en la conformación de la personalidad. Se considera relevante cómo actúan los padres con los gemelos y los mellizos, si es que acentúan diferencias o promueven parecidos. También destaca tener en cuenta que con el ciclo evolutivo aparece la necesidad del parecido y de la diferencia. Una acción educativa deseable en gemelos seria acompañándolos en el proceso de construcción de la imagen de sí mismo, teniendo en cuenta que esta construcción fluctúa entre la necesidad de identificarse con el otro y la necesidad de diferenciarse respecto al otro. (Cerruti et al., 2008).

Las condiciones especiales de la gestación y parto que se desencadenan en las familias múltiples ocasionan una serie de consecuencias nuevas y particulares, que generan inevitablemente ansiedad en toda la familia. El bajo peso al nacer y la prematurez que caracteriza a estos niños demandan directamente mayor cuidado de los padres. (Amar Amar, Pesellín Andrade, y Tirado García, 2010). Durante la gestación, las repre- sentaciones mentales de las madres sobre su embarazo se centran en su papel de cuidadoras de la salud de sus hijos y en la creencia inamovible de ser las encargadas de llevar el embarazo a término. A pesar de saber que las posibilidades de llevar un embarazo múltiple hasta el noveno mes son muy poco probables, en todos los casos estudiados en la investigación de Amar Amar et al., las madres sintieron culpa y ansiedad por parir antes de término. Desde los primeros meses del embarazo sus mayores preocupaciones pasan por una posible lesión o daño en la salud física de sus hijos. Esta condición les obstaculiza crear expectativas de la imagen física, o imaginar el parecido con familiares. Estas representaciones mentales acerca de la maternidad evidencian que el embarazo múltiple genera una disponibilidad ansiosa obsesiva especialmente en las madres. En un principio, el manejo de la situación excede al manejo real de los padres, y estos suelen desarrollar una representación mental de sí mismos como ansiosos, inexpertos, sobrepasados por la vivencia de la multiplicidad. Suelen asociar la imagen de los hijos como débiles, frágiles y demandantes. Las madres se caracterizan por mantener una atención excesiva y ansiosa a las necesidades de los hijos, a tal punto de que pueden llegar al descuido de las propias necesidades o de pareja. Sin em- 
bargo, la experiencia se va a ir adquiriendo a través del contacto y el aprendizaje en los cuidados. La sincronía entre las demandas de los niños y el cuidado adecuado de los padres les permitirán sentirse capaces, disponibles, habilidosos y podrán lograr una aceptación más real de su posición como padres de gemelos. (Amar Amar et al., 2010).

La gemelaridad, además de un suceso biológico, es un constructo que es culturalmente interpretado y vivido de forma distinta por las diferentes sociedades. (Stewart, 2003).

Oliveira Machado y Uchoa Branco (2010), consideran que es esencial conocer lo que los padres reflexionan sobre la gemelaridad. Los sistemas de creencias parentales son constituidos por encuentros entre las experiencias diarias de los padres y a veces con expertos de las vías de la comunicación social. Es relevante tener en cuenta la esencia de esos sistemas de creencias y sus características afectivas, ya que a veces si se está perjudicando el desarrollo infantil de un niño, las emociones parentales pueden obstaculizar la reflexión y la reorientación de valores en caso de que sea necesario. Las creencias cumplen una función organizadora de la mente de las acciones manifestadas por los padres.

Klein (2003), sostiene que el patrón de gemelaridad y el tipo de vínculo entre los gemelos es determinado por la calidad de la educación recibida en la infancia.

Oliveira Machado y Uchoa Branco (2010), intentan responder a la pregunta de cómo debería ser la socialización y la educación de gemelos. En donde es frecuente encontrarse con disparidades entre el contexto familiar y escolar en cuanto a las expectativas del desempeño, comportamiento y relaciones de los gemelos. Klein (2003), señala que la cultura presenta a los gemelos de una manera estereotipada, con expresiones como dupla, copia, idénticos, clon uno del otro o doble problema. Es muy común conservar la creencia de que mantienen entre sí una relación de intimidad muy particular. La expectativa o la deseabilidad social es que los gemelos constantemente comparten todo y permanecen siempre juntos. La competencia entre ellos les sirve para desplegar distintas funciones, según los adolescentes gemelos que consultó, todos se refirieron a la competencia como una articulación de comparación y contraste en donde pueden exhibir sus identidades. La competencia entre ellos puede ser una manifestación positiva de sus diferencias, según el patrón de gemelaridad de cada pareja.

Perlman (2004), postula que los padres múltiples enfrentan el desafío de acompañar a sus hijos gemelos mientras oscilan entre su dependencia e 
independencia. Para el entendimiento de la co-construcción dinámica de las similitudes y diferencias entre gemelos es necesario tener en cuenta la convergencia de las creencias y teorías de los educadores en los distintos contextos de participación social, como la escuela o la familia. Al tener registro de las creencias, uno puede comprender el origen de las expectativas y las prácticas que se ponen en juego en el trascurso de la crianza de gemelos. Es preciso indagar en los patrones de relación, las emociones y las motivaciones que estén implicadas, teniendo en cuenta a todos los participantes de esta dinámica. (Oliveira Machado y Uchoa Branco, 2010).

La manera en que los padres traten a sus gemelos es crucial para el desarrollo de su identidad. No producirán el mismo efecto aquellos padres que los traten como una unidad, que aquellos padres que perciban la impor- tancia de distinguir a sus hijos idénticos como individuos. El desarrollo individual de cada gemelo colabora a que su vínculo sea saludable. Observarlos como individuos y explorar con ellos cuál es el mayor interés de cada uno, aumenta la posibilidad de tomar decisiones saludables sobre ellos. La forma en que los padres ayudan a sus gemelos a desarrollar su propia identidad debe hacerse dependiendo de cada niño. (Perlman, 2004).

Oliveira Machado y Uchoa Branco (2010), afirman que no se puede asegurar que los padres a través de las vivencias que estos proporcionan sean los exclusivos responsables de la individuación y desarrollo de sus hijos gemelos. Los componentes propios de la genética, los presentes en la cultura, las experiencias propias y las características individuales de estos niños intervendrán en su desarrollo y su constitución subjetiva.

\section{TRASTORNOS DE ALIMENTACIÓN Y GEMELAZGO}

La teoría genética señala que es de una a cinco veces más probable que los parientes de primer grado de los pacientes que sufran algún trastorno, sufran el mismo. La heredabilidad en gemelos es de un 50-75\% más. Se cree que la comorbilidad es probablemente debido a factores genéticos que influyen en el riesgo para ciertos trastornos, y además, las teorías psicosociales agregan que la comorbilidad también puede ser causada por sucesos vitales y factores estresantes. (Carol, 2010). Bulik, Sullivan, Tozzi, Furberg, Lichtenstein y Pedersen (2006), estudiaron a los gemelos y encontraron que surge un fuerte 
componente genético superpuesto con factores ambientales en el riesgo de desarrollar anorexia o bulimia.

La tasa de concordancia de los gemelos se postula en torno al 50-55 \%, mientras que la de los mellizos está en torno al 7 \% (Virginia Twin Study de Kendler en 1995), siendo que en más de la mitad de los gemelos que comparten la misma dotación genética, cuando un gemelo desarrolla un trastorno alimentario, el otro también lo padece. (Carol, 2010).

Losada (2011), expresó que la tasa de los trastornos de la conducta alimentaria entre gemelos se constituye mayor que la observada en mellizos, un $44 \%$ versus un $12,5 \%$. El estudio de 34 parejas de gemelos y un caso de trillizos halló que la tasa de anorexia entre gemelos es significativamente mayor que en mellizos. Holland, Hall, Murray, Russell y Crisp, 1984; Wade, Heath, Abraham, Treloar, Martin y Tiggemann, 1996, investigaron acerca de la prevalencia de anorexia y bulimia entre parejas de gemelos en donde se encontró una concordancia del $55 \%$ entre gemelos y patología alimentaria. Analizaron los períodos de 1988 a 1989 y 1992 a 1993. No se encontraron diferencias entre los dos cohortes y la prevalencia para la anorexia fue del 0,4\% y para la bulimia del 1,8\%.

Dentro de las patologías alimen- tarias, la anorexia es ocho veces más común en personas que tienen parientes con ese trastorno. Respecto a esto, Lenoir y Silber (2006), manifiestan su acuerdo en que existe un mayor riesgo de anorexia entre familiares de primer grado. La tasa de concordancia entre gemelos es significativamente mayor que en mellizos. Staudt, Rojo, y Ojeda (2006), coinciden en que los factores genéticos son fundamentales en el desarrollo de la anorexia ya que según ellos la tasa de concordancia es del $70 \%$ para gemelos y del $20 \%$ para los mellizos.

La investigación de Yao, Kuja-Halkola, Thornton, Birgegard, Norring, Bulik y Larsson (2019), reportó correlaciones genéticas significativas, tanto en anorexia como en bulimia nerviosa y el gemelazgo.

Las tasas de concordancia del $52 \%$ al $56 \%$ de trastornos alimentarios en gemelos monocigotos frente a dicigotos con unas tasas entre el 5-11\%, han sido ponderadas y destacadas como contribuciones genéticas frente a estas patologías. (Holland et al., 1984; Holland et al., 1988 citado en Bosque Garza y Caballero Romo, 2008; Wade et al., 2000).

Félix López, Nachón García y Hernández Parra (2003, destacaron concordancias del $44 \%$ entre gemelos, mientras un $12,5 \%$ en mellizos. 


\section{RESILIENCIA}

Para preservar la salud integral de las personas es necesario fortalecer los factores de protección y prevenir los de riesgo. Los factores de protección son descritos por Cornellái Canals y Llusent Guillamet (1999), como los recursos personales o sociales que atenúan o neutralizan el impacto del riesgo. Estos factores son influencias que transforman, mejoran o alteran la respuesta de una persona a algún peligro que predispone a un resultado no adaptativo.

La resiliencia es un factor relevante no solo en beneficiar la calidad de vida sino también como un factor destacado en la resistencia del estrés. Quesada (2003), destaca que la resiliencia puede devenir de factores externos e internos estudiando los atributos del propio niño y comenzaron a estudiar las características de sus familias y del contexto social.

El factor protector más relevante para el desarrollo de las personas correspondería al vínculo afectivo, a las vivencias afectivas y valores vividos en su espacio de crecimiento familiar. La resiliencia se establece como un factor protector a largo plazo. (Losada, 2011; Losada y Marmo, 2017; Losada y Marmo, 2019).

La familia es el sistema de pertenencia más próximo en donde se vinculan las personas. Este sistema es la base del desarrollo y de la socialización. Al ser uno de los organismos de socialización más relevantes para los hijos de cualquier edad, los padres influyen directamente en la configuración de la conducta de los hijos al realizar sus funciones correspondientes. Dentro de cada familia hay factores positivos y negativos que intervienen en el desarrollo de los hijos. Conocer el patrón de crianza, sus creencias y valores actuales que usan los padres es esencial ya que eso representa cómo es el proceso de desarrollo y socialización de los niños. Las prácticas de crianza son las tendencias generales de comportamiento, las prácticas más frecuentes, en donde dentro de un continuum relativamente abundante de tácticas, eligen con elasticidad las pautas educativas. Estas prácticas son diferentes entre los padres, como también sus consecuencias en los hijos. A través de estas prácticas de crianza los padres pretenden formar y dirigir el comportamiento de sus hijos según la dirección que valoran y estiman de acuerdo con su personalidad. Estas prácticas inevitablemente se ven atravesadas por la clase de disciplina, la manifestación de afecto, el tono de la relación y el nivel de comunicación. La crianza es multidimensional ya que incluye conductas específicas que trabajan en forma individual y conjunta con el fin de influir en el niño. Hay tres procesos que participan 
en la crianza: a) las prácticas, que son las acciones, b) las pautas, que son las normas, los patrones y las costumbres y $c$ ) las creencias, como prejuicios, valores y mitos. Este conjunto de dimensiones determinará las expectativas y la forma de desarrollarse. (Luna, 1999).

A través del apoyo afectivo los padres brindan soporte social y alientan la independencia y el control personal de sus hijos. Se relaciona con la expresión del afecto hacia sus hijos y el apoyo para que puedan expresar de forma libre sus emociones. Este apoyo afectivo generalmente se manifiesta a través de caricias, verbalizaciones de afecto, proximidad física, juegos, entre otros. Pudiendo ir desde lo afectuoso como elogios, alabanzas, aprobación, estimulación-aliento, cooperación, términos cariñosos, ternura, afecto físico, ayuda y cooperación hasta lo más distante. La afectividad moderada o apoyo, se refiere al grado en que los padres intencionalmente incentivan la individualidad, la asertividad y la autorregulación, para armonizar el apoyo y la aprobación de las necesidades y requerimientos especiales de los niños. Según Aguirre (2002), el surgimiento de la confianza en sí mismo es facilitado por el contacto físico, permitiendo que se dé de manera más natural la manifestación de afecto en los niños. El apoyo es la conducta que expresa un padre hacia el hijo, que hace que este último se sienta cómodo en presencia del padre y confirme en su mente que es aceptado como un individuo. Es esencial esta manifestación de expresión emocional para que se dé la aceptación del otro y el propio reconocimiento. Las familias funcionales ponen en evidencia que el afecto es uno de los valores más preciados que aporta el entorno, expresando sentimientos positivos como la ternura, el amor, la confianza, el cariño, el apoyo, la admiración, la lealtad y la empatía.

Aguirre Forero (2010), relaciona la resiliencia con variables personales, con el entorno donde creció el niño y el tipo de estrés al cual es sometido. Para ciertos investigadores la resiliencia individual y la colectiva son complementarias ya que la habilidad de enfrentar dificultades y salir airoso incluye respuestas que se puedan dar de manera individual y colectiva. La resiliencia familiar, según Quesada (2003), es la capacidad de auto reparación de una familia. En donde la familia identifica y promueve procesos que le permitan enfrentar crisis con más efectividad y a la vez salir fortalecidas de estas. Está relacionada con el funcionamiento y el desempeño del sistema a lo largo del tiempo, según vayan atravesando los diversos períodos del ciclo vital. La familia está conformada por una estructura, recursos, limitaciones y demandas psicosociales. Si con estos elementos se puede consolidar como un núcleo de re- 
siliencia funcional, puede permear a sus miembros de aquella capacidad. Los factores que favorecen la resiliencia familiar son la ternura, la cohesión, la preocupación por los niños y el vínculo emocional con uno de los padres u otra persona importante que los proteja de un medio vulnerable. Otro grupo de factores protectores son la posibilidad de contar con apoyo externo, límites esclarecidos y ambiente educacional amplio. El desarrollo de intereses y vínculos afectivos es considerado como otro grupo protector en el que la inclusión de intereses y personas importantes ajenas a la familia también fomentan conductas resilientes en situaciones familiares difíciles. El anteúltimo factor es poder establecer una buena relación con pares, y el último factor es haber experimentado vivencias de autoconfianza, de autoeficacia y tener una autoimagen positiva.

Desde el enfoque sistémico los elementos básicos para determinar la resiliencia familiar son la flexibilidad, los procesos de cohesión, la resolución de problemas, la comunicación franca y la reafirmación del sistema de creencias. Desde un enfoque ecológico multidimensional, cada familia se forma por la combinación y superposición de distintos elementos singulares como la vida de sus miembros, la estructura de su familia de origen, la religión, la etnicidad, la posición socioeconómica, los roles asociados al género y la etapa del ciclo vital que está atravesando. Tener presente cómo las familias cohabitan en simultáneo en todos estos contextos, permite evaluar sus recursos y limitaciones. Todas las familias tienen el potencial de ser resilientes de distintas maneras. Desde las teorías del estrés se considera crucial la conciencia subjetiva que tienen las familias sobre sus habilidades para manejar ciertas situaciones. Las creencias comunes de una familia conforman y refuerzan las pautas de interacción de sus miembros, determinando la forma en que cada familia encauce situaciones nuevas. (Quesada, 2003).

Fonagy, Steele, Steele, Higgitt, y Target (1994); Suárez Ojeda (1993); Suárez-Ojeda y Krauskopf (1995) y Werrner (1993), describen el funcionamiento psicológico de los niños resilientes que los protege del estrés, señalando las particularidades que se hacen presentes como tener habilidades de resolución de problemas, motivación al logro autogestionado, mayor coeficiente intelectual, mejores estilos de afrontamiento, empatía, manejo adecuado de relaciones interpersonales, autonomía, locus de control interno, capacidad de planificación, voluntad, y sentido del humor positivo. Rutter (1993) y Poseck, Carbelo Baquero y Vecina (2006), destacan como factores resilientes mayor autoestima y tendencia al acercamiento. El sostén social, que 
actúa como un recurso del entorno y la capacidad para solucionar problemas sociales, que es un recurso propio, mejoran el funcionamiento de los niños. Cuando el apoyo social no es suficiente, las personas pueden cubrir sus necesidades buscando otras alternativas. Para Rutter (1999), su concepción de resiliencia abarca la diversidad individual en cómo las personas a lo largo del tiempo responden a los riesgos. Para Kirby y Fraser (1997), el riesgo y la resiliencia forman un continuo, en donde cada constructo encarna el contrario del otro. Grotberg (1995), señala que la resiliencia se presenta como una aptitud universal que le permite a una persona, una familia o un grupo minimizar o sobreponerse de las dificultades o anticiparse a adversidades inexcusables. Las conductas resilientes pueden ser: sostener la calma, mantener el desarrollo normal, o impulsar el crecimiento individual a pesar del presente que se esté atravesando.

Peñacoba y Moreno (1998) y Carpenter (2002), describen los factores protectores que promueven comportamientos resilientes en niños, observando que es necesario pero no determinante poseer una mayor inteligencia y habilidad de resolución de problemas. Así también, el integrar el género femenino lo consideran como una variable protectora, a raíz de observar mayor fragilidad al riesgo en los hombres. Block, Block y
Morrison (1981), demostraron que frente al estrés infantil, la calidad de las interacciones parentales genera más consecuencias en el funcionamiento psicológico de los niños que en el de las niñas. Shaffer (1999), afirma que los niños al ser más vulnerables necesitan un ambiente más organizado y predecible. Los vínculos afectivos externos y el desarrollo de intereses, pudiendo contar con una persona significativa fuera del núcleo familiar favorecen el desarrollo de comportamientos resilientes en situaciones familiares adversas. A la vez el apego parental, logrando entablar una relación afectuosa, cálida y de apoyo con al menos uno de los padres, aplaca los efectos dañinos de un medio desfavorable. El buen temperamento y la buena conducta, como la relación con pares, distingue a los niños no resilientes, por tener una relación de mejor calidad con sus pares.

Aguirre Forero (2010), destaca como factores protectores el autoconcepto y autoevaluación, autoestima, autonomía, empatía, humor y creatividad. Estos rasgos no están exentos de ser influidos por variables que se mencionaron anteriormente como los procesos de la crianza. El autoconcepto según González-Pienda, Núñez Pérez, Glez-Pumariega, y García García (1997), se entiende como la imagen que uno posee de sí mismo determinada por la integración de la información externa e interna, la 
cual es juzgada por la forma específica que tiene el sujeto de razonar y seleccionada en ciertos aspectos significativos por la afectividad. La autoestima es la valoración de sí mismo que tiene un niño en base a sentimientos e ideas que se originan de su propio autoconocimiento, de influencias o mensajes de otras personas y de su medio sociocultural. Tener una autoestima adecuada posibilita afrontar y recuperarse de las adversidades, la noción de las propias capacidades permiten notificar el dolor que desencadenan las situaciones de fracaso. (Salgado Lévano, 2009). La autonomía es la capacidad de tomar decisiones y accionar independientemente de acuerdo a los propios intereses y posibilidades según el momento del desarrollo. Decidiendo conscientemente qué reglas son las que van a dirigir su comportamiento. Es el fruto de la interacción de componentes cognitivos y afectivos que van progresando a medida que evoluciona el desarrollo. Salgado Lévano (2009), la define también como la competencia de inferir pensamientos y sentimientos de otros, pudiendo generar así sentimientos de comprensión, ternura y simpatía.

El humor, según Wolin y Wolin (1993), es la inclinación del espíritu a la alegría. Posibilita apartarse del foco de conflicto, positiviza, relativiza, y permite elaborar de forma lúdica, encontrar lo chistoso entre la tragedia; definido como la capacidad de manifestar palabras, expresiones corporales o faciales que contengan elementos incoherentes y divertidos que resulten tranquilizadores y placenteros. Salgado Lévano (2004), describe que la relación entre la resiliencia y el sentido del humor se manifiesta a través de la tolerancia madura de los fracasos, la ternura por lo defectuoso, la confianza cuando las cosas no salen bien, aquello que posibilita reconocer el sufrimiento y el dolor y convierte la situación adversa en algo más soportable y positivo.

La creatividad es a partir del caos y el desorden, la capacidad de crear orden, belleza y metas, apreciando los problemas desde una perspectiva nueva y dando soluciones novedosas y eficaces. (Wolin y Wolin, 1993). Salgado Lévano (2004), vincula este rasgo con la resiliencia, dando a entender que la creatividad permite considerar las situaciones y los problemas adversos desde diversos ángulos. Poniendo en marcha los propios recursos se pueden llegar a dar soluciones novedosas y originales.

Losada y Latour (2012), definen la resiliencia como aquella capacidad de los seres humanos que les permite sobreponerse a la adversidad y construir sobre ella. La consecuencia es la adaptación positiva producto de un proceso dinámico, aun en contextos de gran adversidad. En todo ser humano está la posibilidad de 
desarrollar la resiliencia en diversos grados. Los elementos protectores o resilientes forman como una coraza para que el individuo se pueda ir defendiendo de las agresiones y limitaciones del medio en que le toca vivir. Un sujeto resiliente cuen- ta con habilidad, adaptabilidad, baja susceptibilidad, desafío efectivo, capacidad, resistencia a la destrucción, conductas vitales positivas, temperamento especial y habilidades cognitivas, las cuales se ejercitan frente a contextos vitales adversos.

\section{DISCUSIÓN}

Al estudiar la elevada concordancia que existe entre la condición de ser gemelo y padecer un trastorno de la conducta alimentaria del tipo de anorexia y bulimia, en este trabajo se intentó comprender si la resiliencia pudiera actuar como factor protector para evitar el desarrollo del trastorno en cuestión.

En los trastornos de la conducta alimentaria se hallan implicados factores biológicos y genéticos, socioculturales, familiares y psicológicos que intervienen en su origen o que pueden influir en su mantenimiento.

Este trabajo se delimitó a estudiar los trastornos de la conducta alimentaria presentes en gemelos, donde la tasa de concordancia se sitúa en torno a más del cincuenta por ciento. Esto significa que en más de la mitad de los gemelos, cuando uno de ellos desarrolla un trastorno alimentario el otro también lo padece. La genética puede predisponer a que una persona sea más propensa a padecer cierto trastorno; sin embargo, puede ocurrir que el trastorno no se presente a pesar de esta predisposición genética. Los estudios analizados han señalado que el trastorno de la conducta alimentaria se despliega por un fuerte componente genético superpuesto con factores ambientales. Es este el motivo por el cual se profundizó el análisis de los factores ambientales que explicarían por qué gemelos que crecieron en un ambiente familiar similar, pueden diferenciarse respecto a su conducta alimentaria, siendo patológica en unos casos mientras que en otros no.

La manera en la que un gemelo se moviliza desde una posición de nosotros a una posición de yo es arduo trabajo de la niñez y continúa en la adultez. Si este proceso ya es dificultoso para cualquier ser humano, será aún más para los gemelos por tener un compañero constante que es idéntico, del cual deben aprender a distinguirse. Y en la medida que la apariencia sea exacta, el proceso es ampliamente más difícil. Los trastor- 
nos de la alimentación se presentan con mayor frecuencia en la adolescencia, momento en el que un sujeto atraviesa un proceso de construcción de la identidad, cambio y crecimiento. Cuanto más perturbado afectivamente se encuentre un adolescente menos tolerante será con su estado físico y por ende será más susceptible a presentar un trastorno de la conducta alimentaria.

No es posible asegurar que los padres a través de las vivencias que estos proporcionan sean responsables de la individuación y desarrollo de sus hijos gemelos, pero sí pueden promover la constitución de una familia con características resilientes que cuente con la capacidad de generar determinados procesos que le permitan enfrentar dificultades con mayor efectividad y a la vez salir fortalecidas de estas. La resiliencia individual y la colectiva son complementarias ya que la habilidad de enfrentar dificultades y salir airoso incluye respuestas que se puedan dar de manera individual y colectiva. La familia es el sistema de pertenencia más próximo en donde se vinculan las personas, base del desarrollo y de la socialización. Los padres al llevar adelante su rol parental influyen directamente en la configuración de la conducta de los hijos.

Las condiciones de crianza que se desencadenan en las familias múltiples ocasionan una serie de consecuencias nuevas y particulares que generan inevitablemente ansiedad en toda la familia. En un principio, el manejo de la situación excede al manejo real de los padres y estos suelen desarrollar una representación mental de sí mismos como ansiosos, inexpertos, sobrepasados por la vivencia de la multiplicidad.

Los elementos de identidad, como el sentimiento de identidad y el sentido de separación se entreveran en la familia, la matriz de la identidad. Es en los primeros procesos de socialización, donde las familias van moldeando y programando la conducta del niño y el sentido de la identidad. El sentido de pertenencia va acompañando de una acomodación del niño a los grupos familiares y su aceptación de pautas transaccionales en la estructura familiar, que se mantendrán a través de los diferentes acontecimientos de la vida. El sentido de pertenencia a una familia específica influye directamente en el sentido de la identidad de cada miembro.

En el proceso de organización de sí mismos los gemelos tienen que reconocerse y distinguirse inclusive con su propio co-gemelo. La construcción de la imagen de sí mismo está entrelazada por la separación y por la distinción del propio gemelo. Este trayecto psíquico es imprescindible para poder llegar a percibirse como un individuo singular y provisto de una identidad autónoma. 
Cómo reflexionen los padres acerca de la gemelaridad será la base de las prácticas educativas, socializadoras y características de los gemelos. El modo en que los padres traten a sus gemelos es crucial para el desarrollo de su identidad. No producirán el mismo efecto aquellos padres que los traten como una unidad, caracterizada por una extrema codependencia y la no individualización entre ellos, que aquellos padres que perciban la importancia de distinguir a sus hijos idénticos como individuos, en donde los padres respondan de forma positiva a las diferencias reales entre los hijos, enfatizando la individualidad y respetando su unión favoreciendo el desarrollo de vínculos íntimos, seguros y por fuera de la díada.

La forma en que los padres ayudan a sus gemelos a desarrollar su propia identidad debe hacerse dependiendo de cada niño. Dilucidar cuál es el interés de cada uno aumenta la posibilidad de tomar decisiones saludables sobre ellos.

Es necesario tener en cuenta que tanto los procesos imitativos e identificativos como los de diferenciación, son vitales y cruciales en la conformación de la personalidad. Para acompañarlos de la mejor manera posible hay que tener en cuenta que en su proceso de construcción de la imagen de sí mismo necesitan fluctuar entre la necesidad de identificarse con el otro y la necesidad de diferenciarse respecto al otro. La calidad de la educación recibida en la infancia va a determinar el tipo de vínculo entre los gemelos. Es crucial el desarrollo individual de cada gemelo para que su vínculo sea saludable.

La familia es el primer grupo de pertenencia donde se transmiten actitudes y creencias y se modelan conductas alimentarias que incorporará el sujeto a lo largo de su vida. Esta puede propiciar la aparición de trastornos y puede mantenerlos. Un contexto familiar negativo predispone mayor vulnerabilidad y menor protección social. Las familias afectadas por este trastorno presentan menor flexibilidad, cohesión, comunicación intrafamiliar, expresión emocional, mayor rechazo y sobreprotección paterna. Las pacientes perciben que en su familia se les da escasa importancia a las dimensiones del desarrollo como la autonomía y las actividades culturales y sociales. El ambiente lo describen deteriorado y existe la presencia de sentimientos negativos entre los familiares. Otro factor de riesgo es la trasmisión cultural que se da dentro de una familia sobre la importancia de la apariencia física. En el seno familiar son riesgosos los comentarios reiterados sobre los cuerpos y siluetas de los adolescentes.

En el funcionamiento familiar la escasa o nula expresión de los sentimientos e intereses, dejando de compartir sentimientos y emociones dentro de las 
familias deja al descubierto necesidades básicas como la aceptación y el afecto. La desorganización como la falta de planificación en las actividades cotidianas y la carencia de asignación de las responsabilidades familiares también se constituye como un factor riesgoso a la hora de desarrollar patologías alimentarias.

En cuanto a lo personal, los principales factores psicológicos que están asociados a los trastornos de la conducta alimentaria son la distorsión de la imagen corporal, la insatisfacción, los índices de masa corporal extremos, la baja autoestima, el afecto negativo, la depresión, la ansiedad, el uso y abuso de dietas injustificadas, ser mujer en un país industrializado, la herencia, la historia familiar de trastornos alimentarios, no poder comunicar emociones negativas con facilidad, ser perfeccionista, ser complaciente con los demás, la excesiva rigidez, tener padres sobreprotectores, no tener habilidades para resolver conflictos, sentirse presionada por el medio, vivir experiencias adversas, tener desbalanceados los neurotransmisores como la serotonina, hacer dieta asociada a presiones individuales, interpersonales y socioculturales. Estos factores preparan el terreno para el desarrollo, arraigo y agravación de estas enfermedades.

La resiliencia es considerada como factor protector frente al riesgo de trastorno de la conducta alimentaria. El factor protector más relevante para el desarrollo de las personas corresponde al vínculo afectivo, a las vivencias afectivas y valores vividos en su espacio de crecimiento familiar. Por el contrario, en el caso del trastorno de la conducta alimentaria al no percibir a sus familias de ese modo, se genera un alto impacto desfavorable.

A través del apoyo afectivo los padres brindan soporte social y alientan la independencia y el control personal de sus hijos. Se relaciona con la expresión del afecto hacia sus hijos y el apoyo para que puedan expresar de forma libre sus emociones. La afectividad permite que los padres intencionalmente incentiven la individualidad, la asertividad y la autorregulación, para armonizar el apoyo y la aprobación de las necesidades y requerimientos especiales de los niños. El apoyo de un padre hacia su hijo hace que este último se sienta cómodo en presencia del padre y confirme en su mente que es aceptado como un individuo. Es esencial esta manifestación de expresión emocional para que se dé la aceptación del otro y el propio reconocimiento. Las familias funcionales ponen en evidencia que el afecto es uno de los valores más preciados que aporta el entorno, expresando sentimientos positivos como la ternura, el amor, la confianza, el cariño, el apoyo, la admiración, la lealtad y la empatía.

Los factores que favorecen la resiliencia familiar son la ternura, la cohe- 
sión, la preocupación por los niños y el vínculo emocional con uno de los padres u otra persona importante que los proteja de un medio vulnerable, la posibilidad de contar con apoyo externo, límites esclarecidos y ambiente educacional amplio, el desarrollo de intereses y vínculos afectivos, establecer una buena relación con pares, haber experimentado vivencias de autoconfianza, de autoeficacia y tener una autoimagen positiva.

Los factores protectores que promueven comportamientos resilientes en niños y aplacan los efectos dañinos de un medio desfavorable constan en poseer una mayor inteligencia y habilidad de resolución de problemas, integrar el género femenino, establecer vínculos afectivos externos y desarrollar intereses, el poder contar con una persona significativa fuera del núcleo familiar, el apego parental en donde se pueda entablar una relación afectuosa, cálida y de apoyo con al menos uno de los padres, buen temperamento, buena conducta y tener una relación de mejor calidad con sus pares. Los rasgos individuales que pueden considerarse resilientes son el autoconcepto, autoevaluación, autoestima, autonomía, empatía, humor y creatividad.

La resiliencia fue identificada en este trabajo como factor protector del trastorno de la conducta alimentaria en gemelos. Es uno de los factores que protege y mantiene la salud y puede encontrarse no solo en las personas sino también en sus interacciones familiares. En este trabajo se ha expuesto cómo los gemelos y las personas que los rodean pueden responder de forma positiva para evitar que desarrollen un trastorno de la conducta alimentaria, teniendo en cuenta tanto las fortalezas y potencialidades de los gemelos como también las características resilientes de una familia que puede favorecer el fortalecimiento y bienestar en la trama social.

\section{FUTURAS LÎNEAS DE INVESTIGACIÓN}

Los resultados de este trabajo reflejan la necesidad de llevar a cabo más estudios epidemiológicos sobre el trastorno de la conducta alimentaria en gemelos. El seguir trabajando con la hipótesis de que el tipo de crianza de los gemelos es un componente clave en la formación de su identidad y que ayudará a que en el transcurso de la adolescencia cada gemelo lo procese lo más fortalecido posible. Mucho es lo que habría que aprender de las familias resilientes para las intervenciones en grupos familiares que atraviesan situaciones de riesgo. 


\section{REFERENCIAS}

Aguirre Forero, A. M. (2010.) Prácticas de crianza y su relación con rasgos resilientes de niños y niñas. Tesis de maestría no publicada, Universidad Nacional de Colombia, Bogotá.

Aguirre, E. (2002). Prácticas de crianza y pobreza. Revista Diálogos: Discusiones en la psicología contemporánea, (2).

Amar Amar, J. J., Pesellín Andrade, M., y Tirado García, D. (2010). Vínculo de apego que establecen padres con hijos nacidos de embarazos múltiples. Revista Científica Salud Uninorte, 25(2).

Ballard, C. K., Bricker, L., Reed, K., Wood, L., Neilson, J. P., Ballard, C. y Neilson, J. (2011). Asesoramiento nutricional para mejorar los resultados en los embarazos múltiples. Cochrane Database of Systematic Reviews, 6.

Bank, S. P. y Kahn, M. D. (1988). El Vínculo fraterno. Barcelona: Paidos.

Block, J. H., Block, J. y Morrison, A. (1981) Acuerdo y desacuerdo de los padres en la crianza de los niños y las correlaciones de personalidad sobre el sexo de los niños. Child Development, 52, 965-974.

Bosque-Garza, J.M., y Caballero-Romo, A. (2009). Consideraciones psiquiá- tricas de los trastornos de la conducta alimentaria: anorexia y bulimia. Boletín médico del Hospital Infantil de México, 66 (5), 398-409.

Bulik, C.M., Sullivan, P.F., Tozzi, F., Furberg, H., Lichtenstein, P. y Pedersen, N.L. (2006). Prevalence, heritability, and prospective risk factors for anorexia nervosa. Archives of General Psychiatry, 63, 305-312.

Calvo, R. (2002). Anorexia y bulimia: guía para padres, educadores y terapeutas. Barcelona: Planeta.

Carol, A. S. (2010). Comorbilidad en los trastornos de la conducta alimentaria. Psicología clínica y de la salud ISEP, 1-61.

Carpenter, B. (2002). Inside the portrait of a family: the importance of fatherhood. Early Child Development and Care, 172(2),195-202.

Castro, J. y Toro, J. (2004). Anorexia Nerviosa: el peligro de adelgazar. Barcelona: Morales Torres.

Cerruti, G., Prino, L. E. y Quaglia, R. (2008) La imagen de sí mismos que tienen los niños gemelos a través de los dibujos. Infad Revista de Psicología, 1, 91-100.

Coccaro, M. I. (2012) Prevalencia de Trastornos de la Conducta Alimentaria en alumnos de 12 a 18 años en escuelas de la ciudad de La Plata. 
Provincia de Buenos Aires. Argentina. Tesis de postgrado, Universidad Nacional de La Plata, Buenos Aires.

Cornellái Canals, J. y Llusent Guillamet, À. (1999). Aspectos generales de la salud en la adolescencia y la juventud: Factores de riesgo y de protección. Estudios de Juventud, 47, 39-44.

Cruz-Coke, R. (1980). Fundamentos genéticos del comienzo de la vida humana. Revista chilena de pediatría, 51(2), 121-124.

Durier, M., Vervaet, H. y Gabriel, R. (2010). Embarazos múltiples. Estudio anatomoclínico y tratamiento. EMC-Ginecología-Obstetricia, 46(4), 1-12.

Dyer, J. y McGuinness, T. (1996). Resilience: Analysis of the concept. Archive of Psychiatric Nursing, 10, 276-282.

Eddleman, K.A. , Stone, J. L., Lynch, L. \& Berkowitz, R. L. (2002). Selective termination of anomalous fetuses in multifetal pregnancies: two hundred cases at a single center. Am J Obstet Gynecol; 87, 1168.

Endres, L. \& Wilkins, L. (2005). Epidemiology and biology of multiples gestations. Clin Perinatol, 32, 301-314.

Félix López, G.A., Nachón García, M.G., y Hernández Parra, T.G. (2003). Anorexia nerviosa: una revisión.
Psicología y Salud, 13 (1), 121-133. Fernández García, A., Gálvez González, A. y Villar Valdés, R. (2014). Fundamentos de los estudios de costo de la enfermedad: valoración actual del costo del glaucoma. Revista Cubana de Medicina General Integral 26(3).

Fonagy, P., Steele, M., Steele, H., Higgitt, A. y Target M.(1994). The Emanuel Miller Memorial Lecture 1992. The theoryand practice of resilience. Journal of Child Psychology and Psychiatry. 35(2), 231-258.

Fraser, M. W. (1997). Risk and resilience in childhood: An ecological perspective. Washington, DC: Nasw press.

González, L., Hidalgo, M., Hurtado, M., Nova, C. y Venegas, M. (2011). Relación entre factores individuales y familiares de riesgo para desórdenes alimenticios en alumnos de enseñanza media. Revista de Psicología, 11(1),91.

González-Pienda, J. A., Pérez, J. C. N., Pumariega, S. G., y García, M. S. G. (1997). Autoconcepto, autoestima y aprendizaje escolar. Psicothema, 9(2), 271-289.

Gromada, K. K. y Hurlburt, M. C. (2006). Dos o más: claves para la crianza de gemelos, trillizos o más. Bogotá. Editorial Norma. (PLAN)

Grotberg, E.H. (1995). The international resilience project: Research, appli- 
cation, and policy. Recuperado de http://eric.ed.gov/?id=ED423955. Guirao Goris, J. A., Olmedo Salas, A., y Ferrer Ferrandis, E. (2008). El artículo de Revisión. Revista Iberoamericana de Enfermería Comunitaria, 1(1), 1-25.

Herscovici, C. R. y Bay, L. (1990). Aspectos generales en Anorexia Nerviosa y Bulimia. Amenazas a la autonomía. Barcelona: Paidós.

Holland, A. J., Hall, A., Murray, R., Russell, G. F., y Crisp, A. H. (1984). Anorexia nervosa: a study of 34 twin pairs and one set of triplets. The British Journal of Psychiatry, 145(4), 414419.

Kancyper, L. (2004). El complejo fraterno. Buenos Aires: Grupo Editorial Lumen.

Kirby, L.D. y Fraser, M.W. (1997). Risk and resilience in childhood: An ecological perspective. Washington, DC: Nasw Press. (PLAN)

Klein, B. S. (2003). Not all twins are alike: Psychological profiles of twinship. Westport: Praeger. (PLAN)

Laterra, C., Susacasa, S., Frailuna, M. A., Flores, L. y Secondi, V. (2006). Embarazo doble: diez años de resultados perinatales en el Hospital Materno Infantil Ramón Sardá (1992-2001). Revista Hospital Materno Infantil Ramón Sarda, 25(1), 32-37.
Lenoir, M. y Silber, T. J. (2006). Anorexia nerviosa en niños y adolescentes: criterios diagnósticos, historia, epidemiología, etiología, fisiopatología, morbilidad y mortalidad. Archivos argentinos de pediatría, 104(3), 253-260.

Losada, A. V. (2011). Abuso sexual infantil y patologías alimentarias. Tesis de Doctorado, Universidad Católica Argentina. Buenos Aires.

Losada, A. V., y Latour, M. I. (2012). Resiliencia. Conceptualización e investigaciones en Argentina. PSIENCIA. Revista Latinoamericana de Ciencia Psicológica, 4(2).

Losada, A. V. (2015). Familia y psicología. Buenos Aires: Dunken.

Losada, A. V. y Marmo, J. (2017). Hacia la comprensión de los procesos familiares. Buenos Aires: Dunken.

Losada, A. V. y Marmo, J. (2019). Manual de Psicología de la familia. Buenos Aires: Educa.

Luna, M. T. (1999) Prácticas de crianza en Antioquia. Un estudio en Familias Campesinas. Colombia, Medellín: Cinde.

Mahler, M. S., Pine, F., y Bergman, A. (1977). El nacimiento psicológico del infante humano: simbiosis e individuación. Buenos Aires: Marymar.

Mares, M. y Casanueva, E. (2001). Embarazo gemelar. Determinantes ma- 
ternas del peso al nacer. Perinatol Reprod Hum, 15(4), 238-244.

Minuchin, S. (1997). Familia y terapia familiar. Buenos Aires: Gedisa.

Oliveira Machado, A. y Uchoa Branco, A. (2010). Iguales, pero diferentes: creencias sociales en la canalización cultural del desarrollo de gemelos. Revista de Psicología, 28 (2.)

Peñacoba C. y Moreno, B. (1998) El concepto de personalidad resistente: Consideraciones teóricas y repercusiones prácticas. Boletín de Psicología, 58, 61-96.

Perlman, L. (2004). ¿Yo soy "yo" o soy "nosotros"? Ayudando a los gemelos a ser individuos. Recuperado de http://www.multifamilias.org. ar/twins4.pdf.

Poseck, V., Carbelo, B., y Vecina, M. (2006). La experiencia traumática desde la psicología positiva: resiliencia y crecimiento postraumático. Papeles del psicólogo, 27(1), 40-49.

Quesada, C. V. (2003). El concepto de resiliencia individual y familiar. Aplicaciones en la intervención social. Intervención Psicosocial, 12(3), 283-299.

Rodríguez Remón, A. (2013). Papel de la familia en los trastornos de la conducta alimentaria. Recuperado de http://zaguan.unizar.es/ TAZ/SOCIZ/2013/12011/TAZ-
TFG-2013-751.pdf

Rutter, M. (1993). Resilience: Some Conceptual Considerations. Journal of Adolescent Health, 14(8), 626-631.

Rutter, M. (1999). Resilience concepts and findings: Implications for family therapy. Journal of Family Therapy, 21, 119-144.

Sánchez Ramírez, N., Carrillo Bermúdez, L., Sanabria Arias, A. M., \& Olivella Mejía, L. (2019). Reducción embrionaria en embarazo gemelar. Revista Cubana de Obstetricia y Ginecología, 45(2).

Salgado Lévano, A. C. (2004) Métodos e Instrumentos para medir la resiliencia: una alternativa peruana. Liberabit 11, 41-48.

Salgado Lévano, A. C. (2009) Felicidad, Resiliencia y Optimismo en estudiantes de colegios nacionales en Lima. Liberabit 15(2), 133-141.

Shaffer, D.R. (1999). Psicología del desarro/10, infancia y adolescencia ( $5^{\circ}$ ed.). Georgia: Internacional Thomson Editores.

Staudt, M. A., Rojo, N. M. y Ojeda, G. A. (2006). Trastornos de la conducta alimentaria: anorexia nerviosa. Revisión bibliográfica. Revista de Posgrado de la VI Cátedra de Medicina, 156, 24-30.

Stewart, E. A. (2003). Exploring twins: Towards a social analysis of twinship. Nueva York: Palgrave Macmillan. 
Suárez Ojeda E.N. y Krauskopf, D. (1995). El enfoque de riesgo y su aplicación a las conductas del adolescente: Una perspectiva psico-social. Publicación Científica No. 552, OPS/ OMS. Washington, D.C., EE.UU.

Suárez Ojeda, E. (1993). Resiliencia, O capacidad de sobreponerse a la adversidad. Medicina y sociedad. 16(3), 18-20.

Trias, T., Ebeling, H. E y Moilanen, I. (2012). Relaciones entre gemelos: revisión de literatura. Revista de Psicopatología y salud mental del niño y del adolescente, (20), 47-56.

Vázquez-Arévalo, R., Mancilla-Díaz, J. M., López-Aguilar, X., Álvarez-Rayón, G. G. L.y Tena-Suck, A. (2010). Funcionamiento familiar en el riesgo y la protección de trastornos del comportamiento alimentario; Family functioning in therisk and protection of eatingdisorders. Univ. psychol, 9(2), 447-455.

Wade, T., Heath, AC, Abraham, S., Treloar, SA, Martin, NG y Tiggemann, M. (1996). Evaluación de la prevalencia de trastornos alimentarios en una población gemela australiana. Australian \& New Zealand Journal of Psychiatry , 30 (6), 845851.

Wimalasundera, R. C. (2010). Selective reduction and termination of multiple pregnancies. Semin Fetal Neonatal Med;15:27.

Wolin, S. J. y Wolin, S. (1993) The Resilient Self: How Survivors of Troubled Families Rise above Adversity. Nueva York: Villard.

Yao, S., Kuja-Halkola, R., Thornton, L., Birgegard, A., Norring, C., Bulik, C., \& Larsson, H. (2019). Overlap between anorexia and bulimia nervosa: understanding genetic andenvironmental effects in a swedish population registry. European Neuropsychopharmacology, 29, S817-S818.

Zamora, R., Martinez, A., Gazzo. C. y Cordero, S. (2002) Antecedentes personales y familiares de los trastornos de la conducta alimentaria. Archivos de Pediatría del Uruguay, 73(3), 129-136. 